\title{
TRIANGULATED CATEGORIES OF SINGULARITIES AND EQUIVALENCES BETWEEN LANDAU-GINZBURG MODELS
}

\author{
DMITRI ORLOV
}

\begin{abstract}
In this paper we prove an existence of some type of equivalences between triangulated categories of singularities for varieties of different dimensions. This class of equivalences generalizes so called Knörrer periodicity. As consequence we get equivalences between categories of D-branes of type B on Landau-Ginzburg models of different dimensions.
\end{abstract}

\section{INTRODUCTION}

The bounded derived category of coherent sheaves $\mathbf{D}^{b}(\operatorname{coh}(X))$ is a natural triangulated category which can be attached to an algebraic variety $X$. This category has a triangulated subcategory $\mathfrak{P e r f}(X)$ formed by perfect complexes. A notion of a perfect complex was introduced in [1, and, by definition, it is a complex of sheaves of $\mathcal{O}_{X}$-modules which locally is quasi-isomorphic to a bounded complex of locally free sheaves of finite type (a good reference is [20]).

If the variety $X$ is smooth then any coherent sheaf has a finite resolution of locally free sheaves of finite type and the subcategory of perfect complexes coincides with $\mathbf{D}^{b}(\operatorname{coh}(X))$. But for singular varieties this property is not fulfilled. One can define a triangulated category of singularities $\mathbf{D}_{\mathrm{Sg}}(X)$ as the quotient of the triangulated category $\mathbf{D}^{b}(\operatorname{coh}(X))$ by the full triangulated subcategory of perfect complexes $\mathfrak{P e r f}(X)$ [18. The category $\mathbf{D}_{\mathrm{Sg}}(X)$ reflects the properties of the singularities of $X$ and "does not depend on all of $X$ ". For example it is invariant with respect to a localization in Zariski topology ([18, and Prop. 1.3]).

The investigation of such categories is not only connected with a study of singularities but is mainly inspired by the Homological Mirror Symmetry Conjecture [14.

The HMSC has dealings with Calabi-Yau varieties endowed with symplectic forms. It asserts that if two Calabi-Yau varieties $X$ and $Y$ are mirror symmetric to each other, then the category of D-branes of type B on $X$ is equivalent to the category of D-branes of type A on $Y$, and vice versa. From the mathematical point of view the category of D-branes of type $\mathrm{B}$ on a variety is the derived category of coherent sheaves on it 14, 4. As a candidate for a category of A-branes on a symplectic manifolds the so-called Fukaya category has been proposed. Its objects are, roughly speaking, Lagrangian submanifolds equipped with flat vector bundles [14.

On the other hand, physicists also consider mirror symmetry relation for other varieties for instance Fano varieties. In these cases the so called Landau-Ginzburg theories arise on the mirror

This work is done under partial financial support of the Weyl Fund, the grant RFFI (No 02-01-00468), grant of President of RF in support of young russian scientists MD-2731.2004.1, grant CRDF Award No RM1-2405-MO-02. It is also a pleasure for me to express my gratitude to the Russian Science Support Foundation. 
side [10]. General definition of a Landau-Ginzburg model involves, besides a choice of a target space, a choice of a holomorphic function $W$ on it which is called superpotential.

For Fano varieties one has the derived categories of coherent sheaves (B-branes) and given a symplectic form, one can propose a suitable Fukaya category (A-branes). Thus, if one wants to extend the Homological Mirror Symmetry Conjecture to the non-Calabi-Yau case, one should understand D-branes in Landau-Ginzburg models.

Categories of A-branes in Landau-Ginzburg models are studied in [9] and in [19] from the mathematical point of view. Mirror symmetry relates B-branes on a Fano variety (coherent sheaves) to A-branes in a LG model. One can also consider the Fukaya category (A-branes) on a Fano variety and can expect in this case that the Fukaya category is equivalent to the category of B-branes in the mirror Landau-Ginzburg model.

A mathematical definition for the category of B-branes in Landau-Ginzburg models was proposed by M.Kontsevich. Roughly, he suggests that the superpotential $W$ deforms complexes of coherent sheaves to "twisted" complexes, i.e the composition of differentials is no longer zero, but is equal to multiplication by $W$. In the paper [18] we established a connection between categories of B-branes in Landau-Ginzburg models and triangulated categories of singularities. We considered singular fibres of the map $W$ and showed that the triangulated categories of singularities of these fibres are equivalent to the categories of B-branes. In particular this gives that the category of B-branes depends only on the singular fibres of the superpotential.

In this paper we establish equivalence between Landau-Ginzburg models of different dimensions. More precisely, we prove an equivalence between triangulated categories of singularities of two different schemes. The first one is the zero subscheme of a regular section $s \in H^{0}(S, \mathcal{E})$ of a vector bundle $\mathcal{E}$ on a smooth scheme $S$ and the second one is the respective divisor on the projective bundle $\mathbb{P}\left(\mathcal{E}^{\vee}\right)$ (Theorem 2.1). This result implies an equivalence of categories of Dbranes of type B for different Landau-Ginzburg models. In particular, it says the following: Let $W: Y \rightarrow \mathbb{A}^{1}$ be a superpotential on a variety $Y=S \times \mathbb{A}^{1}$ of the form $W=f+x g$, where $f, g$ are functions on $S$ and $x$ is an coordinate on $\mathbb{A}^{1}$. Then one can reduce dimension and change the Landau-Ginzburg model on $Y$ by the Landau-Ginzburg model on $X \subset S$, where $X$ is the zero subvariety of the function $g$, with a restriction of $f$ as a superpotential. Thus, we prove that the category of B-branes on the Landau-Ginzburg model on $Y$ is equivalent to the category of B-branes in the Landau-Ginzburg model on $X$ (Corollary 3.2). Of course, we expect that the categories of D-branes of type A are also equivalent for these LG-modles.

I am grateful to Anton Kapustin and Ludmil Katzarkov for useful discussions. I also thank to Valery Lunts for reading of a preliminary draft of the paper and making a number of valuable comments. I also would like to express my gratitude to Alexander Kuznetsov who pointed out an existence of semiorthognal decomposition which arises in Proposition 2.10. I want to thank Institute for Advanced Study, where this work was written, for hospitality and very stimulating atmosphere. 


\section{Triangulated CATEgories of Singularities}

We remind that a triangulated category $\mathcal{D}$ is an additive category with the following data:

a) an additive autoequivalence $[1]: \mathcal{D} \longrightarrow \mathcal{D}$ (it is called a translation functor),

b) a class of exact (or distinguished) triangles:

$$
X \stackrel{u}{\longrightarrow} Y \stackrel{v}{\longrightarrow} Z \stackrel{w}{\longrightarrow} X[1]
$$

which must satisfy a certain set of axioms (see [23, also [6, 12, 13, 16]).

A functor $F: \mathcal{D} \longrightarrow \mathcal{D}^{\prime}$ between two triangulated categories $\mathcal{D}$ and $\mathcal{D}^{\prime}$ is called exact if it commutes with the translation functors and transforms exact triangles into exact triangles.

Let $\mathcal{N} \subset \mathcal{D}$ be a full triangulated subcategory, i.e. it is a full subcategory which closed with respect to the translation functor and it is triangulated with respect to exact triangles in $\mathcal{D}$. Denote by $\Sigma(\mathcal{N})$ a class of morphisms $s$ in $\mathcal{D}$ embedding into an exact triangle

$$
X \stackrel{s}{\longrightarrow} Y \longrightarrow N \longrightarrow X[1]
$$

with $N \in \mathcal{N}$. It can be checked that $\Sigma(N)$ is a multiplicative system. Define the quotient $\mathcal{D} / \mathcal{N}$ as a localization $\mathcal{D}\left[\Sigma(\mathcal{N})^{-1}\right]$ [5, 6, 23]. We endow the category $\mathcal{D} / \mathcal{N}$ with a translation functor induced by the translation functor in the category $\mathcal{D}$. The category $\mathcal{D} / \mathcal{N}$ becomes a triangulated category by taking for exact triangles the images of exact triangles in $\mathcal{D}$. The quotient functor $Q: \mathcal{D} \rightarrow \mathcal{D} / \mathcal{N}$ annihilates $\mathcal{N}$. Moreover, any exact functor $F: \mathcal{D} \rightarrow \mathcal{D}^{\prime}$ between triangulated categories for which $F(X) \simeq 0$ when $X \in \mathcal{N}$ factors uniquely through $Q$.

The following lemma, which will be necessary in the future, is evident.

Lemma 1.1. Let $\mathcal{N}$ and $\mathcal{N}^{\prime}$ be full triangulated subcategories of triangulated categories $\mathcal{D}$ and $\mathcal{D}^{\prime}$ respectively. Let $F: \mathcal{D} \rightarrow \mathcal{D}^{\prime}$ and $G: \mathcal{D}^{\prime} \rightarrow \mathcal{D}$ be adjoint pair of exact functors such that $F(\mathcal{N}) \subset \mathcal{N}^{\prime}$ and $G\left(\mathcal{N}^{\prime}\right) \subset \mathcal{N}$. Then they induce functors

$$
\bar{F}: \mathcal{D} / \mathcal{N} \longrightarrow \mathcal{D}^{\prime} / \mathcal{N}^{\prime}, \quad \bar{G}: \mathcal{D}^{\prime} / \mathcal{N}^{\prime} \longrightarrow \mathcal{D} / \mathcal{N}
$$

which are adjoint too. Moreover, if the functor $F: \mathcal{D} \rightarrow \mathcal{D}^{\prime}$ is fully faithful then the functor $\bar{F}: \mathcal{D} / \mathcal{N} \longrightarrow \mathcal{D}^{\prime} / \mathcal{N}^{\prime}$ is fully faithful too.

We are mainly interested in triangulated categories and their quotient by triangulated subcategories which are coming from algebraic geometry.

Let $X$ be a scheme over field $k$. We say that $X$ satisfies the condition (ELF) if it is separated noetherian of finite Krull dimension and has enough locally free sheaves,

(ELF) i.e. for any coherent sheaf $\mathcal{F}$ there is an epimorphism $\mathcal{E} \rightarrow \mathcal{F}$ for a locally free sheaf $\mathcal{E}$.

For example, any quasi-projective scheme satisfies these conditions.

Denote by $\mathbf{D}^{b}(\operatorname{coh}(X))$ (resp. $\quad \mathbf{D}^{b}(\mathrm{Q} \operatorname{coh}(X))$ ) the bounded derived categories of coherent (resp. quasi-coherent) sheaves on $X$. Since $X$ is noetherian the natural functor $\mathbf{D}^{b}(\operatorname{coh}(X)) \longrightarrow$ $\mathbf{D}^{b}(\mathrm{Q} \operatorname{coh}(X))$ is fully faithful and realizes an equivalence of $\mathbf{D}^{b}(\operatorname{coh}(X))$ with the full subcategory 
$\mathbf{D}^{b}(\mathrm{Q} \operatorname{coh}(X))_{\operatorname{coh}} \subset \mathbf{D}^{b}(\mathrm{Q} \operatorname{coh}(X))$ consisting of all complexes with coherent cohomologies (see [1] II, 2.2.2).

The objects of the category $\mathbf{D}^{b}(\operatorname{coh}(X))$ which are isomorphic to bounded complexes of locally free sheaves on $X$ form a full triangulated subcategory. It is called the subcategory of perfect complexes and denoted by $\mathfrak{P e r f}(X){ }^{1}$

Definition 1.2. We define a triangulated category $\mathbf{D}_{\mathrm{Sg}}(X)$ as the quotient of the triangulated category $\mathbf{D}^{b}(\operatorname{coh}(X))$ by the full triangulated subcategory $\mathfrak{P e r f}(X)$ and call it a triangulated category of singularities of $X$.

It is known that if our scheme $X$ is regular then the subcategory of perfect complexes coincides with the whole bounded derived category of coherent sheaves. In this case the triangulated category of singularities is trivial.

Let $f: X \rightarrow Y$ be a morphism of finite Tor-dimension (for example a flat morphism or a regular closed embedding). It defines the inverse image functor $\mathbf{L} f^{*}: \mathbf{D}^{b}(\operatorname{coh}(Y)) \longrightarrow \mathbf{D}^{b}(\operatorname{coh}(X))$. It is clear that the functor $\mathbf{L} f^{*}$ sends perfect complexes on $Y$ to perfect complexes on $X$. Therefore, the functor $\mathbf{L} f^{*}$ induces an exact functor $\mathbf{L} \bar{f}^{*}: \mathbf{D}_{\mathrm{Sg}}(Y) \longrightarrow \mathbf{D}_{\mathrm{Sg}}(X)$.

Suppose, in addition, that the morphism $f: X \rightarrow Y$ is proper and locally of finite type. Then the functor of direct image $\mathbf{R} f_{*}: \mathbf{D}^{b}(\operatorname{coh}(X)) \rightarrow \mathbf{D}^{b}(\operatorname{coh}(Y))$ takes perfect complexes on $X$ to perfect complexes on $Y$ (see [1] III, or [20]). Hence it determines a functor $\mathbf{R} \bar{f}_{*}: \mathbf{D}_{\mathrm{Sg}}(X) \rightarrow$ $\mathbf{D}_{\mathrm{Sg}}(Y)$ which is the right adjoint to $\mathbf{L} \bar{f}^{*}$.

A fundamental property of triangulated categories of singularities is a property of locality.

Proposition 1.3. 18 Let $X$ satisfy (ELF) and let $j: U \hookrightarrow X$ be an embedding of an open subscheme such that $\operatorname{Sing}(X) \subset U$. Then the functor $\bar{j}^{*}: \mathbf{D}_{\mathrm{Sg}}(X) \longrightarrow \mathbf{D}_{\mathrm{Sg}}(U)$ is an equivalence of triangulated categories.

Remark 1.4. The definition of triangulated categories of singularities can be extended to orbifolds and more generally to stacks. We briefly consider the example of quotient stacks. Let $G$ be an affine group scheme of finite type which acts on a scheme $S$ which satisfy (ELF). Consider the quotient stack $[S / G]$. The category of coherent sheaves on this stack $\operatorname{coh}([S / G])$ coincides with the category $\operatorname{coh}^{G}(S)$ of $G$-equivariant coherent sheaves on $S$. The quotient of the bounded derived category of coherent sheaves $\mathbf{D}^{b}(\operatorname{coh}([S / G]))$ by the triangulated subcategory of perfect complexes $\mathfrak{P e r f}([S / G])$, which is formed by bounded complexes of locally free sheaves in $\mathbf{D}^{b}(\operatorname{coh}([S / G])) \cong \mathbf{D}^{b}\left(\operatorname{coh}^{G}(S)\right)$, can be called a triangulated category of singularities of the quotient stack $[S / G]$. (For more information when the quotient stack has enough locally free sheaves see papers [21, 22].)

\footnotetext{
${ }^{1}$ Actually, a perfect complex is defined as a complex of $\mathcal{O}_{X}$-modules locally quasi-isomorphic to a bounded complex of locally free sheaves of finite type. But under our assumption on the scheme any such complex is quasiisomorphic to a bounded complex of locally free sheaves of finite type(see [1] II, or [20] §2).
} 
Remark 1.5. One also can consider noncommutative case. Let $A$ be a right noetherian algebra. Denote by $\bmod -A$ and $\operatorname{Mod}-A$ the abelian categories of finitely generated right modules and all right modules respectively. Consider bounded derived categories $\mathbf{D}^{b}(\bmod -A)$ and $\mathbf{D}^{b}(\operatorname{Mod}-A)$. They have triangulated subcategories consisting of objects which are isomorphic to bounded complexes of projectives. These subcategories can be considered as derived of exact categories of projective modules $\mathbf{D}^{b}(\operatorname{proj}-A)$ and $\mathbf{D}^{b}(\operatorname{Proj}-A)$ respectively (see for example [13]). Now we can define triangulated categories of singularities $\mathbf{D}_{\mathrm{Sg}}(A)$ and $\mathbf{D}_{\mathrm{Sg}}^{\prime}(A)$ as the quotients $\mathbf{D}^{b}(\bmod -A) / \mathbf{D}^{b}(\operatorname{proj}-A)$ and $\mathbf{D}^{b}(\operatorname{Mod}-A) / \mathbf{D}^{b}(\operatorname{Proj}-A)$ respectively. As in the commutative case, if $A$ has a finite homological dimension then these quotient categories are trivial.

There is a way to generalize Definition 1.2 to any triangulated category. Let $\mathcal{D}$ be a triangulated category.

Definition 1.6. We say that an object $A$ is homologically finite if for any object $B \in \mathcal{D}$ all $\operatorname{Hom}(A, B[i])$ are trivial except for finite number of $i \in \mathbb{Z}$. All such objects form a full triangulated subcategory which will be denoted by $\mathcal{D}_{\mathrm{hf}}$.

Definition 1.7. We define a triangulated category $\mathcal{D}_{\mathrm{Sg}}$ as the quotient $\mathcal{D} / \mathcal{D}_{\mathrm{hf}}$ of the triangulated category $\mathcal{D}$ by the full triangulated subcategory $\mathcal{D}_{\mathrm{hf}}$.

These categories $\mathcal{D}_{\mathrm{hf}}$ and $\mathcal{D}_{\mathrm{Sg}}$ have good behavior with respect to semiorthogonal decomposition of $\mathcal{D}$. We recall some definitions and facts concerning admissible subcategories and semiorthogonal decompositions (see [2, 3]).

Definition 1.8. Let $I: \mathcal{N} \hookrightarrow \mathcal{D}$ be an embedding of a full triangulated subcategory $\mathcal{N}$ in a triangulated category $\mathcal{D}$. We say that $\mathcal{N}$ is right admissible (resp. left admissible) if there is a right (resp. left) adjoint functor $P: \mathcal{D} \rightarrow \mathcal{N}$. The subcategory $\mathcal{N}$ will be called admissible if it is right and left admissible.

Let $\mathcal{N} \subset \mathcal{D}$ be a full triangulated subcategory. The right orthogonal to $\mathcal{N}$ is a full subcategory $\mathcal{N}^{\perp} \subset \mathcal{D}$ consisting of all objects $M$ such that $\operatorname{Hom}(N, M)=0$ for any $N \in \mathcal{N}$. The left orthogonal ${ }^{\perp} \mathcal{N}$ is defined analogously. The orthogonals are also triangulated subcategories.

The property to be right admissible for the subcategory $\mathcal{N}$ is equivalent to the following: for each $X \in \mathcal{D}$ there is an exact triangle $N \rightarrow X \rightarrow M$, where $N \in \mathcal{N}$ and $M \in \mathcal{N}^{\perp}$.

If $\mathcal{N} \subset \mathcal{D}$ is an admissible subcategory then we say that the category $\mathcal{D}$ has semiorthogonal decompositions of the form $\left\langle\mathcal{N}^{\perp}, \mathcal{N}\right\rangle$ and $\left\langle\mathcal{N},{ }^{\perp} \mathcal{N}\right\rangle$.

Definition 1.9. A sequence of admissible subcategories $\left(\mathcal{N}_{1}, \ldots, \mathcal{N}_{n}\right)$ in a derived category $\mathcal{D}$ is said to be semiorthogonal if the condition $\mathcal{N}_{j} \subset \mathcal{N}_{i}^{\perp}$ holds when $j<i$ for any $1 \leq i \leq n$. In addition, a semiorthogonal sequence is said to be full if it generates the category $\mathcal{D}$. In this case we call it as a semiorthogonal decomposition of the category $\mathcal{D}$ and denote this as

$$
\mathcal{D}=\left\langle\mathcal{N}_{1}, \ldots, \mathcal{N}_{n}\right\rangle
$$


Proposition 1.10. Suppose that a triangulated category $\mathcal{D}$ has a semiorthogonal decomposition $\mathcal{D}=\left\langle\mathcal{N}_{1}, \ldots, \mathcal{N}_{n}\right\rangle$. Then the categories $\mathcal{D}_{\mathrm{hf}}$ and $\mathcal{D}_{\mathrm{Sg}}$ also have semiorthogonal decompositions of the same form

$$
\mathcal{D}_{\mathrm{hf}}=\left\langle\left(\mathcal{N}_{1}\right)_{\mathrm{hf}}, \ldots,\left(\mathcal{N}_{n}\right)_{\mathrm{hf}}\right\rangle, \quad \mathcal{D}_{\mathrm{Sg}}=\left\langle\left(\mathcal{N}_{1}\right)_{\mathrm{Sg}}, \ldots,\left(\mathcal{N}_{n}\right)_{\mathrm{Sg}}\right\rangle .
$$

Proof. First, note that if a functor $u: \mathcal{D} \longrightarrow \mathcal{D}^{\prime}$ has a right adjoint $v: \mathcal{D}^{\prime} \longrightarrow D$ then $u\left(\mathcal{D}_{\mathrm{hf}}\right) \subset \mathcal{D}^{\prime}{ }_{\mathrm{hf}}$, because

$$
\operatorname{Hom}(u A, B[j]) \cong \operatorname{Hom}(A, v B[j])
$$

for any $A \in \mathcal{D}, B \in \mathcal{D}^{\prime}$. Moreover, if $u$ is a full embedding then $u\left(\mathcal{D}_{\mathrm{hf}}\right)=\mathcal{D}^{\prime}{ }_{\mathrm{hf}} \cap u(\mathcal{D})$.

Second, we can assume that $n=2$. Since $\mathcal{N}_{k}$ are admissible the embedding functor $i_{k}$ : $\mathcal{N}_{k} \longrightarrow \mathcal{D}$ has right adjoint $p_{k}$. Hence, it takes any homologically finite object to a homologically finite object. Now suppose $X \in \mathcal{D}_{\mathrm{hf}}$ and consider decomposition

$$
i_{1} p_{1}(X) \longrightarrow X \longrightarrow i_{2} q_{2}(X),
$$

where $q_{2}: \mathcal{D} \longrightarrow \mathcal{N}_{2}$ is left adjoint to $i_{2}$. As we proved above $q_{2}(X) \in\left(\mathcal{N}_{2}\right)_{\text {hf }}$. Hence, $i_{2} q_{2}(X) \in \mathcal{D}_{\mathrm{hf}}$. This implies that $i_{1} p_{1}(X) \in \mathcal{D}_{\mathrm{hf}}$ and, consequently, $p_{1}(X) \in\left(\mathcal{N}_{1}\right)_{\mathrm{hf}}$. This gives us the semiorthogonal decomposition for $\mathcal{D}_{\mathrm{hf}}$ of the form (11). Finally, applying Lemma 1.1 we obtain the semiorthogonal decomposition for $\mathcal{D}_{\mathrm{Sg}}$ of the form $\mathcal{D}_{\mathrm{Sg}}=\left\langle\left(\mathcal{N}_{1}\right)_{\mathrm{Sg}}, \ldots,\left(\mathcal{N}_{n}\right)_{\mathrm{Sg}}\right\rangle$.

Proposition 1.11. Let $X$ satisfies (ELF). Then the subcategory $\mathcal{D}_{\mathrm{hf}}$ of homologically finite objects in $\mathcal{D} \cong \mathbf{D}^{b}(\operatorname{coh}(X))$ coincides with the subcategory $\mathfrak{P e r f}(X)$ and, hence, $\mathcal{D}_{\mathrm{Sg}} \cong \mathbf{D}_{\mathrm{Sg}}(X)$.

Proof. If an object $A \in \mathcal{D}$ is a perfect complex then it is quasi-isomorphic to a bounded complex of vector bundles. Since the cohomologies of any coherent sheaf is bounded by the Krull dimension of variety we have that for any vector bundle $\mathcal{P}$ and any coherent sheaf $\mathcal{F}$ there is equality $\operatorname{Ext}^{i}(\mathcal{P}, \mathcal{F})=0$ when $i$ is greater than Krull dimension of $X$. Therefore, $A$ belongs to the subcategory $\mathcal{D}_{\mathrm{hf}}$.

Suppose now that $A \in \mathcal{D}_{\text {hf }}$. The object $A$ is a bounded complex of coherent sheaves. Let us take locally free bounded above resolution $P \stackrel{\sim}{\rightarrow} A$ and consider a good truncation $\tau^{\geq-k} P^{\cdot}$ for sufficient large $k \gg 0$ which is clearly isomorphic to $A$ in $\mathcal{D}$.

Since $A \in \mathcal{D}_{\mathrm{hf}}$ for any closed point $t: x \hookrightarrow X$ the groups $\operatorname{Hom}\left(A, t_{*} \mathcal{O}_{x}[i]\right)$ are zero for $|i| \gg 0$. This means that for sufficiently large $k \gg 0$ the truncation $\tau^{\geq-k} P$ is a complex of locally free sheaves at the point $x$, and, hence, in some neighborhood of $x$. The scheme $X$ is quasicompact. This implies that there is a common sufficiently large $k$ such that the truncation $\tau^{\geq-k} P^{r}$ is a complex of locally free sheaves everywhere on $X$, i.e. $A$ is perfect.

Corollary 1.12. Let $X$ satisfies (ELF). Suppose that the bounded derived category of coherent sheaves on $X$ has a semiorthogonal decomposition $\mathbf{D}^{b}(\operatorname{coh}(X))=\left\langle\mathcal{D}_{1}, \ldots, \mathcal{D}_{n}\right\rangle$. Then the triangulated category of singularities $\mathbf{D}_{\mathrm{Sg}}(X)$ has the semiothogonal decomposition of the form

$$
\mathbf{D}_{\mathrm{Sg}}(X)=\left\langle\left(\mathcal{D}_{1}\right)_{\mathrm{Sg}}, \ldots,\left(\mathcal{D}_{n}\right)_{\mathrm{Sg}}\right\rangle .
$$


Example 1.13. Let $X$ be a projective space bundle $\mathbb{P}(\mathcal{E})$, where $\mathcal{E}$ is a vector bundle of rank $r$ on a scheme $Y$. It can be shown that the bounded derived category $\mathbf{D}^{b}(\operatorname{coh}(X))$ has a semiorthogonal decomposition of the form

$$
\mathbf{D}^{b}(\operatorname{coh}(X))=\left\langle p^{*} \mathbf{D}^{b}(\operatorname{coh}(Y)) \otimes \mathcal{O}(i), \ldots, p^{*} \mathbf{D}^{b}(\operatorname{coh}(Y)) \otimes \mathcal{O}(i+r-1)\right\rangle,
$$

for any $i \in \mathbb{Z}$, where $p: X \longrightarrow Y$ is the projection. This statement for a smooth base can be found in [17] and the proof works for any base. By Corollary 1.12 the subcategory of perfect complexes $\mathfrak{P e r f}(X)$ also has the semiorthogonal decomposition of the same form

$$
\mathfrak{P e r f}(X) \cong\left\langle p^{*} \mathfrak{P e r f}(Y) \otimes \mathcal{O}(i), \ldots, p^{*} \mathfrak{P e r f}(Y) \otimes \mathcal{O}(i+r-1)\right\rangle .
$$

And, finally, we obtain the semiorthogonal decomposition for the triangulated category of singularities of $X$ in the terms of the triangulated category of singularities of $Y$.

Triangulated categories of singularities of $X$ have an additional good properties in case of scheme is Gorenstein. Recall that a local noetherian ring $A$ is called Gorenstein if $A$ as a module over itself has a finite injective resolution. It can be shown that if $A$ is Gorenstein than $A$ is a dualizing complex for itself (see [8]). This means that $A$ has finite injective dimension and the natural map

$$
M \longrightarrow \mathbf{R} \operatorname{Hom}^{*}\left(\mathbf{R} \operatorname{Hom}^{*}(M, A), A\right)
$$

is an isomorphism for any coherent $A$-module $M$ and, as a consequence, for any object from $\mathbf{D}^{b}(\operatorname{coh}(\operatorname{Spec}(A)))$.

Definition 1.14. A scheme $X$ is Gorenstein if all of its local rings are Gorenstein local rings.

If $X$ is Gorenstein and has finite dimension, then $\mathcal{O}_{X}$ is a dualizing complex for $X$, i.e. it has finite injective dimension as the quasi-coherent sheaf and the natural map

$$
\mathcal{F} \longrightarrow \mathbf{R} \underline{\mathcal{H o m}}\left(\mathbf{R} \underline{\mathcal{H} o m}\left(\mathcal{F}, \mathcal{O}_{X}\right), \mathcal{O}_{X}\right)
$$

is an isomorphism for any coherent sheaf $\mathcal{F}$. In particular, there is an integer $n_{0}$ such that $\underline{\mathcal{E} x t^{i}}\left(\mathcal{F}, \mathcal{O}_{X}\right)=0$ for each quasi-coherent sheaf $\mathcal{F}$ and all $i>n_{0}$.

The following statements and their proofs can be found in [18].

Lemma-Definition 1.15. Let $X$ satisfy (ELF) and be Gorenstein. We say that a coherent sheaf $\mathcal{F}$ is Cohen-Macaulay if the following equivalent conditions hold.

1) The sheaves $\underline{\mathcal{E} x t^{i}}\left(\mathcal{F}, \mathcal{O}_{X}\right)$ are trivial for all $i>0$.

2) There is a right locally free resolution $0 \longrightarrow \mathcal{F} \longrightarrow\left\{Q^{0} \longrightarrow Q^{1} \longrightarrow Q^{3} \longrightarrow \cdots\right\}$.

Lemma 1.16. Let $X$ satisfy (ELF) and be Gorenstein. Let $\mathcal{F}$ be a Cohen Macaulay sheaf which is perfect as a complex. Then $\mathcal{F}$ is locally free.

Proposition 1.17. Let $X$ satisfy (ELF) and be Gorenstein. Then any object $A \in \mathbf{D}_{\mathrm{Sg}}(X)$ is isomorphic to the image of a Cohen-Macaulay sheaf $\mathcal{F}$. 


\section{Reduction of Dimension}

Let $S$ be a separated regular noetherian scheme of finite Krull dimension. Let $\mathcal{E}$ be a vector bundle on $S$ of rank $r$ and let $s \in H^{0}(S, \mathcal{E})$ be a section. Denote by $X \subset S$ the zero subscheme of $s$. We will assume that the section $s$ is regular, i.e. the codimension of the subscheme $X$ coincides with the rank $r^{2}$. In particular, the restriction of the bundle $\mathcal{E}$ on $X$ coincides with the normal bundle to $X$ in $S$ which will be denoted by $\mathcal{N}_{X / S}$

Consider the associated projective space bundles $\mathbb{P}\left(\mathcal{E}^{\vee}\right)$ and $\mathbb{P}\left(\mathcal{N}_{X / S}^{\vee}\right)$, where $\mathcal{E}^{\vee}$ and $\mathcal{N}_{X / S}^{\vee}$ are the dual vector bundles. Denote these schemes by $S^{\prime}$ and $Z$ respectively, and the projections onto $S$ and $X$ by $q$ and $p$ respectively. There are canonical line bundles on $S^{\prime}$ and $Z$, denoted by $\mathcal{O}_{\mathcal{E}}(1)$ and $\mathcal{O}_{\mathcal{N}}(1)$, respectively, and the canonical surjections

$$
q^{*} \mathcal{E} \longrightarrow \mathcal{O}_{\mathcal{E}}(1), \quad p^{*} \mathcal{N}_{X / S} \longrightarrow \mathcal{O}_{\mathcal{N}}(1)
$$

The section $s$ induces a section $w \in H^{0}\left(S^{\prime}, \mathcal{O}_{\mathcal{E}}(1)\right)$. Denote by $Y$ the zero divisor on $S^{\prime}$ defined by the section $w$. The natural closed embedding of $Z=\mathbb{P}\left(\mathcal{N}_{X / Y}\right)$ into $S^{\prime}$ goes through $Y$. Consider the closed embedding $i: Z \hookrightarrow Y$. The kernel of the latter map of (2) coincides with the normal bundle to $N_{Z / Y}$, i.e. there is an exact sequence of sheaves on $Z$

$$
0 \longrightarrow \mathcal{N}_{Z / Y} \longrightarrow p^{*} \mathcal{N}_{X / S} \longrightarrow \mathcal{O}_{\mathcal{N}}(1) \longrightarrow 0 .
$$

All schemes defined above can be included in the following commutative diagram.

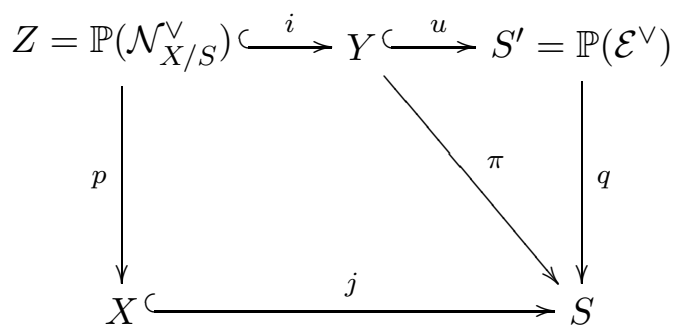

Consider the composition functor $\mathbf{R} i_{*} p^{*}: \mathbf{D}^{b}(\operatorname{coh}(X)) \rightarrow \mathbf{D}^{b}(\operatorname{coh}(Y))$ and denote it by $\Phi_{Z}$. The aim of this section is to prove the following theorem.

Theorem 2.1. Let $S, S^{\prime}, X, Y$, and $Z$ be as above. Then the functor $\Phi_{Z}: \mathbf{D}^{b}(\operatorname{coh}(X)) \rightarrow$ $\mathbf{D}^{b}(\operatorname{coh}(Y))$ defined by the formula $\Phi_{Z}(\cdot)=\mathbf{R} i_{*} p^{*}(\cdot)$ induces a functor

$$
\bar{\Phi}_{Z}: \mathbf{D}_{\mathrm{Sg}}(X) \longrightarrow \mathbf{D}_{\mathrm{Sg}}(Y)
$$

which is an equivalence of triangulated categories.

We give two proofs of this theorem. Both of them use the following proposition.

Proposition 2.2. The functor $\Phi_{Z}=\mathbf{R} i_{*} p^{*}: \mathbf{D}^{b}(\operatorname{coh}(X)) \rightarrow \mathbf{D}^{b}(\operatorname{coh}(Y))$ is fully faithful.

\footnotetext{
${ }^{2}$ By definition, regularity means that the Koszul complex constructed with $s$ is exact, but since $S$ is regular it is equivalent to the codimension of the zero subscheme being the right one.
} 
Proof. First, note that the functor $\Phi_{Z}=\mathbf{R} i_{*} p^{*}$ has a right adjoint functor which we denote by $\Phi_{Z *}$. It can be represented as a composition $\mathbf{R} p_{*} i^{b}$, where $i^{b}$ is right adjoint to $\mathbf{R} i_{*}$ and has the form $\mathbf{L} i^{*}\left(\cdot \otimes \omega_{Z / Y}\right)[-r+1]$, where $\omega_{Z / Y} \cong \Lambda^{r-1} \mathcal{N}_{Z / Y}$ (see, for example, [8] III, Cor.7.3).

Second, one can see that the functor $p^{*}: \mathbf{D}^{b}(\operatorname{coh}(X)) \longrightarrow \mathbf{D}^{b}(\operatorname{coh}(Z))$ is fully faithful, because $\mathbf{R} p_{*} \mathcal{O}_{Z} \cong \mathcal{O}_{X}$ and by the projection formula we obtain isomorphisms

$$
\mathbf{R} p_{*} p^{*}(A) \cong A \otimes \mathbf{R} p_{*} \mathcal{O}_{Z} \cong A \otimes \mathcal{O}_{X} \cong A
$$

for every $A \in \mathbf{D}^{b}(\operatorname{coh}(X))$.

Now consider the canonical transformation of the functors id $\rightarrow \Phi_{Z *} \Phi_{Z}$. To prove the proposition we have to show that this transformation is an isomorphism. This means we should check that for any object $A \in \mathbf{D}^{b}(\operatorname{coh}(X))$ an object $C_{A}$ coming from an exact triangle

$$
A \longrightarrow \Phi_{Z *} \Phi_{Z} A \longrightarrow C_{A}
$$

is isomorphic to the zero object. All objects $A$ for which $C_{A}=0$ form a triangulated subcategory of $\mathbf{D}^{b}(\operatorname{coh}(X))$. Since the minimal triangulated subcategory which contains all sheaves coincides with whole $\mathbf{D}^{b}(\operatorname{coh}(X))$ it is enough to show that $C_{A}=0$ when $A=\mathcal{G}$ is a sheaf.

Let $A=\mathcal{G}$ be a sheaf. Since the functor $p^{*}$ is fully faithful, the triangle (5) is the image of the triangle

$$
p^{*} \mathcal{G} \longrightarrow i^{b} \mathbf{R} i_{*} p^{*} \mathcal{G} \longrightarrow B_{\mathcal{G}}
$$

under the functor $\mathbf{R} p^{*}$, where the first morphism is the canonical map induced by the natural transformation id $\rightarrow i^{b} \mathbf{R} i_{*}$. We have the following sequence of isomorphisms

$$
\begin{array}{r}
\left.\mathbf{R} i_{*}{ }^{b} \mathbf{R} i_{*} p^{*} \mathcal{G} \cong \mathbf{R} i_{*}\left(\mathbf{L} i^{*} \mathbf{R} i_{*} p^{*} \mathcal{G} \otimes \omega_{Z / Y}\right)[-r+1]\right) \cong \mathbf{R} i_{*}\left(p^{*} \mathcal{G} \otimes \omega_{Z / Y}\right) \otimes_{\mathcal{O}_{Y}} \mathcal{O}_{Z}[-r+1] \cong \\
\left.\mathbf{R} i_{*}\left(p^{*} \mathcal{G} \otimes \omega_{Z / Y}\right) \stackrel{\mathbf{L}}{\mathcal{O}_{Z}}\left(\mathcal{O}_{Z} \stackrel{\mathbf{L}}{\otimes_{\mathcal{O}_{Y}}} \mathcal{O}_{Z}\right)[-r+1]\right) .
\end{array}
$$

It is known (see [1] VII, 2.5) that $\underline{\mathcal{T}}$ or $_{k}^{\mathcal{O}_{Y}}\left(\mathcal{O}_{Z}, \mathcal{O}_{Z}\right) \cong \Lambda^{k} \mathcal{N}_{Z / Y}^{\vee}$.

Hence, the object $\mathbf{R} i_{*} i^{b} \mathbf{R} i_{*} p^{*} \mathcal{G}$ is a complex the (r-k-1)-th cohomology of which is isomorphic to $i_{*}\left(p^{*} \mathcal{G} \otimes \omega_{Z / Y} \otimes \Lambda^{k} \mathcal{N}_{Z / Y}^{\vee}\right)$. Therefore, the object $i^{b} \mathbf{R} i_{*} p^{*} \mathcal{G}$ is a complex on $Z$ the $k$-th cohomology of which is isomorphic to $p^{*} \mathcal{G} \otimes \Lambda^{k} \mathcal{N}_{Z / Y}$ for $k=0, \ldots, r-1$. This implies that the object $B_{\mathcal{G}}$ has nontrivial cohomologies only for $k=1, \ldots, r-1$, and $H^{k}\left(B_{\mathcal{G}}\right)$ is isomorphic to $p^{*} \mathcal{G} \otimes \Lambda^{k} \mathcal{N}_{Z / Y}$

Now, applying the functor $\mathbf{R} p_{*}$ to the exact sequence (3) we obtain that $\mathbf{R} p_{*} \mathcal{N}_{Z / Y} \cong 0$. Moreover, it can be easily checked that

$$
\mathbf{R} p_{*} \Lambda^{k} \mathcal{N}_{Z / Y} \cong 0 \quad \text { for } \quad k>0 .
$$

(This is a relative analogue of the fact that on a projective space $H^{i}\left(\Omega^{k}(-k)\right)=0$ for all $i$ and all $k>0$, which is a particular case of the Bott formula.) The equalities (8) implies that

$$
\mathbf{R} p_{*} H^{k}\left(B_{\mathcal{G}}\right) \cong 0 \quad \text { for all } k .
$$


Hence, $\mathbf{R} p_{*} B_{\mathcal{G}}=0$. Thus, the functor $\Phi_{Z}=\mathbf{R} i_{*} p^{*}$ is fully faithful.

It can be useful to note that actually we have proven the following more general proposition.

Proposition 2.3. Let $p: Z=\mathbb{P}\left(\mathcal{N}^{\vee}\right) \rightarrow X$ be the projective space bundle associated to a vector bundle $\mathcal{N}$ on a scheme $X$. Suppose that there is a regular closed embedding $i: Z \hookrightarrow Y$ such that the normal bundle $\mathcal{N}_{Z / Y}$ coincides with the kernel of the canonical map $p^{*} \mathcal{N} \rightarrow \mathcal{O}_{\mathcal{N}}(1)$. Then the composition functor $\mathbf{R} i_{*} p^{*}: \mathbf{D}^{b}(\operatorname{coh}(X)) \longrightarrow \mathbf{D}^{b}(\operatorname{coh}(Y))$ is fully faithful.

Corollary 2.4. The functor $\Phi_{Z}: \mathbf{D}^{b}(\operatorname{coh}(X)) \rightarrow \mathbf{D}^{b}(\operatorname{coh}(Y))$ induces a functor

$$
\bar{\Phi}_{Z}: \mathbf{D}_{\mathrm{Sg}}(X) \longrightarrow \mathbf{D}_{\mathrm{Sg}}(Y)
$$

which is fully faithful too.

Proof. First, the functors $p^{*}$ and $i^{b}=\mathbf{L} i^{*}(\cdot \otimes \mathcal{O}(Z))[-1]$ take perfect complexes to perfect complexes as functors of inverse images. Second, the functors of direct images $\mathbf{R} i_{*}$ and $\mathbf{R} p_{*}$ also preserve perfect complexes, because both morphisms $i$ and $p$ have finite Tor-dimension. Thus, by Lemma 1.1 we get a functor $\bar{\Phi}_{Z}: \mathbf{D}_{\mathrm{Sg}}(X) \rightarrow \mathbf{D}_{\mathrm{Sg}}(Y)$ which is fully faithful.

We constructed the functor $\bar{\Phi}_{Z}: \mathbf{D}_{\mathrm{Sg}}(X) \longrightarrow \mathbf{D}_{\mathrm{Sg}}(Y)$ and showed that this functor is fully faithful.

Now we give the first proof of Theorem 2.1. We will show that any object $F \in \mathbf{D}_{\mathrm{Sg}}(Y)$ satisfying condition $\bar{\Phi}_{Z *} F=0$ is isomorphic to the zero object. The property for $\bar{\Phi}_{Z}$ to be an equivalence is formally deduced from this fact.

The proof is based on the following two simple lemmas.

Lemma 2.5. Let $i: Z \hookrightarrow Y$ be a closed embedding. Let $\mathcal{F}$ be a coherent sheaf on $Y$ such that its restriction to the complement $U=Y \backslash Z$ is locally free and $\mathbf{L} i^{*} \mathcal{F}$ is isomorphic to a locally free sheaf on $Z$. Then $\mathcal{F}$ is locally free on $Y$.

Proof. To prove that $\mathcal{F}$ is locally free it is sufficient to show that for any closed point $t: y \hookrightarrow Y$ we have the equalities $\operatorname{Ext}^{i}\left(\mathcal{F}, t_{*} \mathcal{O}_{y}\right)=0$ for all $i>0$. The sheaf $\mathcal{F}$ is locally free on $U$. Hence, we only need to consider the case $y \in Z$. This means that $t=i \cdot t^{\prime}$ where $t^{\prime}: y \hookrightarrow Z$ is closed embedding. In this case

$$
\operatorname{Ext}_{Y}^{i}\left(\mathcal{F}, t_{*} \mathcal{O}_{y}\right)=\operatorname{Hom}_{Z}^{i}\left(\mathbf{L} i^{*} \mathcal{F}, t_{*}^{\prime} \mathcal{O}_{y}\right)=0
$$

for $i>0$, because $\mathbf{L} i^{*} \mathcal{F}$ is isomorphic to a locally free sheaf on $Z$.

Lemma 2.6. An object $B \in \mathbf{D}^{b}(\operatorname{coh}(Z))$ is perfect if and only if $\mathbf{R} p_{*}(B(n))$ are perfect objects in $\mathbf{D}^{b}(\operatorname{coh}(X))$ for all $n \in \mathbb{Z}$.

Proof. If the objects $B$ is perfect then the objects $\mathbf{R} p_{*}(B(n))$ are perfect too. The inverse statement follows from the semiorthogonal decomposition for $\mathbf{D}^{b}(\operatorname{coh}(Z))$ which was described in Remark 1.13

Remark 2.7. The statement of Lemma 2.6 remains true for any smooth morphism $Z \rightarrow X$. 
Using these two lemmas we can prove the following proposition.

Proposition 2.8. Assume that an object $F \in \mathbf{D}_{\mathrm{Sg}}(Y)$ satisfies the condition $\bar{\Phi}_{Z *} F \cong 0$. Then $F \cong 0$ in $\mathbf{D}_{\mathrm{Sg}}(Y)$.

Proof. At the beginning note that all schemes $X, Z$, and $Y$ are Gorenstein as they are locally complete intersections in regular schemes. By Proposition 1.17 we can assume that the object $F$ is represented by a Cohen-Macaulay sheaf $\mathcal{F}$, in particular $\underline{\mathcal{E} x t^{i}}\left(\mathcal{F}, \mathcal{O}_{Y}\right)=0$ for all $i \neq 0$. Note that any such $\mathcal{F}$ is locally free on the complement $Y \backslash \operatorname{Sing}(Y)$. In addition, for such $\mathcal{F}$ we have $\mathbf{L} i^{*} \mathcal{F} \cong i^{*} \mathcal{F}$ is a sheaf, because $\mathcal{F}$ has a right locally free resolution.

Denote by $\mathcal{L}$ the relatively ample line bundle on $Y$ obtained by the restriction of the line bundle $\mathcal{O}_{\mathcal{E}}(1)$. Consider the object $G=\mathbf{L} u^{*} \mathbf{R} u_{*} \mathcal{F}$, where $u: Y \hookrightarrow S^{\prime}$ is a closed embedding of the divisor $Y$. On the one hand, the object $G$ is a perfect complex, because it is an inverse image of a bounded complex of coherent sheaves from the smooth variety $S^{\prime}$. On the other hand, the complex $G$ has two cohomologies: $\mathcal{F}$ in the zero place and $\mathcal{F} \otimes \mathcal{L}^{-1}$ in the $(-1)$-st place. Thus the image of $\mathcal{F} \otimes \mathcal{L}^{-1}$ in $\mathbf{D}_{\mathrm{Sg}}(Y)$ is isomorphic to the image of $\mathcal{F}[-2]$. By the assumption, the object $\bar{\Phi}_{Z *} \mathcal{F}$ is zero, hence $\bar{\Phi}_{Z *}\left(\mathcal{F} \otimes \mathcal{L}^{n}\right)=0$ for all $n \in \mathbb{Z}$.

Denote by $\mathcal{F}^{\prime}$ the sheaf $i^{*} \mathcal{F}$. We checked that $\mathbf{R} p_{*}\left(\mathcal{F}^{\prime}(n)\right)$ are perfect as complexes on $X$ for all $n \in \mathbb{Z}$. By Lemma 2.6 the sheaf $\mathcal{F}^{\prime}=i^{*} \mathcal{F}$ is perfect as the complex on $Z$.

On the other hand, the sheaf $\mathcal{F}^{\prime}=i^{*} \mathcal{F}$ has a right locally free resolution, i.e. it is also a Cohen-Macaulay sheaf on $Z$. Lemma 1.16 implies now that $\mathcal{F}^{\prime}=i^{*} \mathcal{F}=\mathbf{L} i^{*} \mathcal{F}$ is locally free on $Z$. Therefore, by Lemma 2.5 the sheaf $\mathcal{F}$ is locally free on whole $Y$. This means that $F$ is isomorphic to the zero object in $\mathbf{D}_{\mathrm{Sg}}(Y)$.

The first proof of Theorem 2.1 By Corollary 2.4 we already know that the functor $\bar{\Phi}_{Z}$ is fully faithful. Now let us check that the functor $\bar{\Phi}_{Z *}$ is also fully faithful. Take an object $B \in \mathbf{D}_{\mathrm{Sg}}(Y)$ and consider the natural map $\bar{\Phi}_{Z} \bar{\Phi}_{Z *} B \rightarrow B$. Denote by $C_{B}$ its cone. Applying the functor $\bar{\Phi}_{Z *}$ to the obtained exact triangle and using fully faithfulness of the functor $\bar{\Phi}_{Z}$, we get that $\bar{\Phi}_{Z *} C_{B} \cong 0$. By Proposition 2.8 the object $C_{B}$ is isomorphic to the zero object. Hence, the map $\bar{\Phi}_{Z} \bar{\Phi}_{Z *} B \rightarrow B$ is an isomorphism and the functor $\bar{\Phi}_{Z *}$ is fully faithful.

Remark 2.9. Theorem 2.1 can be generalized to the case of quotient stacks. Suppose that a group scheme $G$ acts on $S$ such that $\mathcal{E}$ can be considered as an equivariant vector bundle with an invariant section $s$. In this case we can extend the $G$-action to all schemes $X, Y, Z$ and $S^{\prime}$. Moreover, we get a functor between equivariant categories $\mathbf{R} i_{*} p^{*}: \mathbf{D}^{b}\left(\operatorname{coh}^{G}(X)\right) \rightarrow \mathbf{D}^{b}\left(\operatorname{coh}^{G}(Y)\right)$. This functor induces a functor $\mathbf{D}_{\mathrm{Sg}}([X / G]) \longrightarrow \mathbf{D}_{\mathrm{Sg}}([Y / G])$ which is an equivalence of triangulated categories as well.

The second proof of Theorem 2.1 is based on a semiorthogonal decomposition of the derived category of coherent sheaves on $Y$. This kind of semiorthogonal decomposition is a particular case of more general situation which will be described in the paper [15]. 
Proposition 2.10. The category $\mathbf{D}^{b}(\operatorname{coh} Y)$ has a semiorthogonal decomposition of the form

$$
\mathbf{D}^{b}(\operatorname{coh} Y)=\left\langle\mathbf{R} i_{*} p^{*} \mathbf{D}^{b}(\operatorname{coh}(X)), \mathbf{L} \pi^{*} \mathbf{D}^{b}(\operatorname{coh}(S)) \otimes \mathcal{L}, \ldots, \mathbf{L} \pi^{*} \mathbf{D}^{b}(\operatorname{coh}(S)) \otimes \mathcal{L}^{r-1}\right\rangle,
$$

where $\mathcal{L}$ is the restriction of $\mathcal{O}_{\mathcal{E}}(1)$ on $Y$.

Proof. First, by Proposition 2.2 the functor $\mathbf{R} i_{*} p^{*}$ is fully faithful. Second, considering the sequence

$$
0 \longrightarrow \mathcal{O}_{\mathcal{E}}(-1) \longrightarrow \mathcal{O}_{S^{\prime}} \longrightarrow u_{*} \mathcal{O}_{Y} \longrightarrow 0 .
$$

Applying the functors $\mathbf{R} q_{*}\left(\mathcal{O}_{\mathcal{E}}(-k) \otimes-\right)$ to it, we obtain that

$$
\mathbf{R} \pi_{*} \mathcal{O}_{Y} \cong \mathbf{R} q_{*} \mathbf{R} u_{*} O_{Y} \cong \mathcal{O}_{S} \quad \text { and } \quad \mathbf{R} \pi_{*} \mathcal{L}^{-k}=0 \quad \text { for all } \quad 0<k \leq r-2
$$

This implies that the functor $\mathbf{L} \pi^{*}: \mathbf{D}^{b}(\operatorname{coh}(S)) \longrightarrow \mathbf{D}^{b}(\operatorname{coh}(Y))$ is fully faithful and the sequence of subcategories $\left(\mathbf{L} \pi^{*} \mathbf{D}^{b}(\operatorname{coh}(S)) \otimes \mathcal{L}, \ldots, \mathbf{L} \pi^{*} \mathbf{D}^{b}(\operatorname{coh}(S)) \otimes \mathcal{L}^{r-1}\right)$ is semiorthogonal.

Third, let $A \in \mathbf{D}^{b}(\operatorname{coh}(X))$ and $B \in \mathbf{D}^{b}(\operatorname{coh}(S))$. We have a sequence of isomorphisms

$$
\begin{array}{r}
\operatorname{Hom}\left(\mathbf{L} \pi^{*} B \otimes \mathcal{L}^{k}, \mathbf{R} i_{*} p^{*} A\right) \cong \operatorname{Hom}\left(\mathbf{L} i^{*}\left(\mathbf{L} \pi^{*} B \otimes \mathcal{L}^{k}\right), p^{*} A\right) \cong \operatorname{Hom}\left(p^{*} \mathbf{L} j^{*} B, p^{*} A(-k)\right) \cong \\
\operatorname{Hom}\left(\mathbf{L} j^{*} B, A \otimes \mathbf{R} p_{*} \mathcal{O}_{\mathcal{N}}(-k)\right)=0 .
\end{array}
$$

And the last equality holds because $\mathbf{R} p_{*} \mathcal{O}_{\mathcal{N}}(-k)=0$ for $0<k \leq r-1$. Thus we obtain the semiorthogonal sequence of subcategories

$$
\left(\mathbf{R} i_{*} p^{*} \mathbf{D}^{b}(\operatorname{coh}(X)), \mathbf{L} \pi^{*} \mathbf{D}^{b}(\operatorname{coh}(S)) \otimes \mathcal{L}, \ldots, \mathbf{L} \pi^{*} \mathbf{D}^{b}(\operatorname{coh}(S)) \otimes \mathcal{L}^{r-1}\right) .
$$

Finally, we should show that this sequence is full. Denote by $\mathcal{C}$ the subcategory of $\mathbf{D}^{b}(\operatorname{coh}(Y))$ which is generated by the sequence (9). As $\mathcal{C}$ is admissible there is a semiorthogonal decomposition $\mathcal{D}=\left\langle\mathcal{C}^{\perp}, \mathcal{C}\right\rangle$. To show that $\mathcal{C}^{\perp}$ is trivial it is sufficient to check that all structure sheaves of closed points belong to $\mathcal{C}$. Consider a closed point $y \in Y$. Denote by $s$ the image $\pi(y) \in S$. The fiber over $s$ is isomorphic to the projective space $\mathbb{P}^{r-2}$ if $s \in S \backslash X$ and it is isomorphic to $\mathbb{P}^{r-1}$ if $s \in X$. In the first case, since $\pi$ is flat over $s$ we have $\mathcal{O}_{Y_{s}} \otimes \mathcal{L}^{k}=\mathbf{L} \pi^{*} \mathcal{O}_{s} \otimes \mathcal{L}^{k}$. These sheaves for $k=1, \ldots, r-1$ belongs to $\mathcal{C}$ and they form an exceptional collection on the projective space $Y_{s}=\mathbb{P}^{r-2}$. Therefore, the skyscraper sheaf $\mathcal{O}_{y}$ belongs to $\mathcal{C}$ too.

In the second case, we know that $\mathbf{R} i_{*} p^{*} \mathcal{O}_{s}=\mathcal{O}_{Y_{s}}$ belongs to $\mathcal{C}$ and the objects $\mathbf{L} \pi^{*} \mathcal{O}_{s} \otimes \mathcal{L}^{k}=$ $\mathbf{L} j^{*} \mathcal{O}_{Y_{s}} \otimes \mathcal{L}^{k}$ also belong to $\mathcal{C}$ for $k=1, \ldots, r-1$. The object $\mathbf{L} \pi^{*} \mathcal{O}_{s} \otimes \mathcal{L}$ is a complex with two cohomologies $\mathcal{O}_{Y_{s}}$ and $\mathcal{O}_{Y_{s}} \otimes \mathcal{L}$. Since $\mathcal{O}_{Y_{s}}$ belongs to $\mathcal{C}$ then $\mathcal{O}_{Y_{s}} \otimes \mathcal{L} \in \mathcal{C}$ too. Iterating this procedure we obtain that $\mathcal{O}_{Y_{s}} \otimes \mathcal{L}^{k} \in \mathcal{C}$ for all $k=0, \ldots, r-1$. Since $Y_{s}=\mathbb{P}^{r-1}$ by the same reason as above we get that $\mathcal{O}_{y} \in \mathcal{C}$ for all $y \in Y$. Thus our semiorthogonal sequence is full.

The second proof of Theorem 2.1 By Corollary [1.12 a semiorthogonal decomposition of the category $\mathbf{D}^{b}(\operatorname{coh}(X))$ induces the semiorthognal decomposition of $\mathbf{D}_{\mathrm{Sg}}(Y)$. Since $S$ is smooth $\mathbf{D}_{\mathrm{Sg}}(S)$ is trivial. Therefore, we get that the category $\mathbf{D}_{\mathrm{Sg}}(Y)$ is equivalent to $\mathbf{D}_{\mathrm{Sg}}(X)$. 


\section{Application to D-Branes in Landau-Ginzburg models.}

By a Landau-Ginzburg model we mean the following data: a smooth variety $X$ with a symplectic Kähler form $\omega$ and a regular nonconstant function $W$ on $X$ which is considered as a flat map $W: X \longrightarrow \mathbb{A}^{1}$ and which should be a symplectic fibration. The function $W$ is called superpotential. Note that for the definition of D-branes of type B a symplectic form is not needed.

A mathematical definition of the categories of D-branes of type B for affine Landau-Ginzburg models was proposed by M.Kontsevich (see [11, 18]). Roughly speaking, he suggests that superpotential $W$ deforms complexes of coherent sheaves to "twisted" complexes, i.e the composition of differentials is no longer zero, but is equal to multiplication by $W$. This "twisting" also breaks $\mathbb{Z}$-grading down to $\mathbb{Z} / 2$-grading. The equivalence of this definition with the physics notion of Bbranes in LG models was verified in the paper [11] in the case of the usual quadratic superpotential and physical arguments were given supporting Kontsevich's proposal for a general superpotential.

It was proved in the paper [18] (Cor. 3.10) that the category of B-branes on a smooth affine $X$ with a superpotential $W$ is equivalent to the product $\prod_{\lambda \in \mathbb{A}^{1}} \mathbf{D}_{\mathrm{Sg}}\left(X_{\lambda}\right)$, where $X_{\lambda}$ is the fiber

over $\lambda \in \mathbb{A}^{1}$, and this product is finite. For non-affine $X$ the category $\prod_{\lambda \in \mathbb{A}^{1}} \mathbf{D}_{\mathrm{Sg}}\left(X_{\lambda}\right)$ can be considered as a definition of the category of D-branes of type B.

Let $S$ be a smooth quasiprojective variety and let $f, g \in H^{0}\left(S, \mathcal{O}_{S}\right)$ be two regular functions. Suppose that the zero divisor $D \subset S$ defined by the function $g$ is smooth and the restriction of $f$ on $D$ is not constant. We can consider $D$ as a Landau-Ginzburg model with superpotential $f_{D}: D \longrightarrow \mathbb{A}^{1}$. Denote by $D_{\lambda}$ the fiber of the map $f_{D}: D \rightarrow \mathbb{A}^{1}$ over a point $\lambda \in \mathbb{A}^{1}$.

Another Landau-Ginzburg model is given by the smooth variety $T=S \times \mathbb{A}^{1}$ and the superpotential $W: T \rightarrow \mathbb{A}^{1}$ defined by the formula $W=f+x g$, where $x$ is a coordinate on $\mathbb{A}^{1}$. Denote by $T_{\lambda}$ the fiber of $W$ over the point $\lambda$. The natural closed embedding of $Z_{\lambda}=D_{\lambda} \times \mathbb{A}^{1}$ into $T$ induces the closed embedding $i_{\lambda}: D_{\lambda} \rightarrow T_{\lambda}$. Consider the functor

$$
\Phi_{Z_{\lambda}}=\mathbf{R} i_{\lambda *} p_{\lambda}^{*}: \mathbf{D}^{b}\left(\operatorname{coh}\left(D_{\lambda}\right)\right) \rightarrow \mathbf{D}^{b}\left(\operatorname{coh}\left(T_{\lambda}\right)\right),
$$

where $p_{\lambda}: Z_{\lambda} \rightarrow D_{\lambda}$ is the natural projection.

Theorem 3.1. The functor $\Phi_{Z_{\lambda}}: \mathbf{D}^{b}\left(\operatorname{coh}\left(D_{\lambda}\right)\right) \rightarrow \mathbf{D}^{b}\left(\operatorname{coh}\left(T_{\lambda}\right)\right)$ induces a functor $\bar{\Phi}_{Z_{\lambda}}:$ $\mathbf{D}_{\mathrm{Sg}}\left(D_{\lambda}\right) \stackrel{\sim}{\longrightarrow} \mathbf{D}_{\mathrm{Sg}}\left(T_{\lambda}\right)$ which is an equivalence of triangulated categories.

Proof. This is particular case of Theorem 2.1. To apply it we consider a trivial two dimensional vector bundle $\mathcal{E}=\mathcal{O}_{S}^{\oplus 2}$ on $S$ and a section $s_{\lambda} \in H^{0}(S, \mathcal{E})$ which is given by the two functions $g, f-\lambda \mathbf{1}$. Thus $D_{\lambda}$ is the zero subvariety of $s_{\lambda}$ and it is the analogue of the scheme $X$ from Theorem 2.1] Applying the construction preceding that theorem we obtain varieties $Z=X \times \mathbb{P}^{1}$ and $Y \subset S \times \mathbb{P}^{1}$ which are compactifications of $Z_{\lambda}$ and $T_{\lambda}$ respectively. By Theorem 2.1] triangulated categories of singularities of $X=D_{\lambda}$ and $Y$ are equivalent. The complement of $T_{\lambda}$ in $Y$ is a relative ample divisor which does not meet $\operatorname{Sing}(Y)=\operatorname{Sing}\left(T_{\lambda}\right)$. Hence, by Proposition $1.3 \quad Y$ and $T_{\lambda}$ also have equivalent triangulated categories of singularities. 
Corollary 3.2. Let $S$ be a smooth quasi-projective variety. Then the Landau-Ginzburg models $f_{D}: D \rightarrow \mathbb{A}^{1}$ and $W: S \times \mathbb{A}^{1} \rightarrow \mathbb{A}^{1}$, where $W=f+x g$ and $D:=\{g=0\} \subset S$ is smooth, have equivalent categories of D-branes of type $B$.

\section{REFERENCES}

[1] Berthelot P., Grothendieck A., Illusie L., Théorie des intersections et théoreme de Riemann-Roch, Springer Lect. Notes in Math., v.225 (1971).

[2] Bondal A., Kapranov M., Representable functors, Serre functors, and mutations, Izv. Akad. Nauk SSSR Ser.Mat. 53 (1989), pp.1183-1205; English transl. in Math. USSR Izv. 35 (1990), pp.519-541.

[3] Bondal A., Orlov D., Semiorthogonal decomposition for algebraic varieties, preprint MPIM 95/15 (1995), arXiv:math.AG/9506012

[4] Douglas M.R., D-branes, categories and N=1 supersymmetry, J.Math.Phys., v.42, 2818 (2001).

[5] Gabriel, P., Zisman, M. Calculus of fractions and homotopy theory, Ergebnisse der Mathematik und ihrer Grenzgebiete, Band 35 Springer-Verlag New York, Inc., New York (1967).

[6] Gelfand S.I., Manin Yu.I., Homological algebra, Algebra V, Encycl. Math. Sci., 38, Springer, Berlin, (1994).

[7] Hartshorne R., Algebraic geometry, Graduate Texts in Mathematics, No. 52. Springer-Verlag, New YorkHeidelberg, (1977).

[8] Hartshorne R., Residues and Duality, Springer Lect. Notes Math., v.20 (1966).

[9] Hori K., Iqbal A., Vafa C., D-branes and Mirror Symmetry, arXiv:hep-th/0005247

[10] Hori K., Vafa C., Mirror Symmetry, arXiv:hep-th/0002222

[11] Kapustin A., Li Yi, D-branes in Landau-Ginzburg models and algebraic geometry, J. High Energy Phys., JHEP 12 (2003) 005, arXiv:hep-th/0210296

[12] Kashiwara M., Shapira P., Sheaves on Manifolds, Grundlehren 292, Springer-verlag, Berlin Heidelberg, (1990).

[13] Keller B., Derived categories and their uses, Chapter of the Handbook of algebra, Vol. 1, edited by M. Hazewinkel, Elsevier (1996).

[14] Kontsevich M., Homological algebra of mirror symmetry, Proceedings of ICM (Zurich, 1994), 120-139, Basel: Birkhäuser (1995).

[15] Kuznetsov A., Homological projective duality, in preparation.

[16] Neeman A., Triangulated categories, Ann. of Math. Studies, 148. Princeton University Press (2001).

[17] Orlov D., Projective bundles, monoidal transformations and derived categories of coherent sheaves, Izv. Akad. Nauk SSSR Ser.Mat. 56 (1992), 852-862; English transl. in Math. USSR Izv. 38 (1993), 133-141.

[18] Orlov D., Triangulated categories of singularities and D-branes in Landau-Ginzburg models, Trudy Steklov Math. Institute, vol. 246 (2004) pp.200-224.

[19] Seidel P., Vanishing cycles and mutations, Proc. 3rd European Congress of Mathematics (Barcelona, 2000), Vol. II, Progr. Math., 202, Birkhäuser, Basel, (2001), 65-85, arXiv:math.SG/0007115

[20] Thomason R.W., Trobaugh T., Higher Algebraic K-Theory of Schemes and of Derived Categories, The Grothendieck Festschrift v.III, Birhäuser, Boston, Basel, Berlin, (1990), 247-436.

[21] Thomason R.W., Equivariant resolution, linearization, and Hilbert's fourteenth problem over arbitrary base schemes, Adv. in Math., v. 65 (1987), no. 1, 16-34.

[22] Totaro B., The resolution property for schemes and stacks, J. Reine Angew. Math., v.577 (2004), 1-22.

[23] Verdier J.L., Categories derivées, in SGA 4 1/2, Lecture Notes in Math., v.569, Springer-Verlag, (1977).

Algebra Section, Steklov Mathematical Institute RAS, Gubkin str. 8, Moscow 119991, RUSSiA

E-mail address: orlov@mi.ras.ru 